\title{
On the Uniqueness of Barycentric Coordinates
}

\author{
Joe Warren
}

\begin{abstract}
Given a convex polytope $P$, a set of coordinate functions attached to the vertices of $P$ is barycentric if the functions are non-negative on $P$ and reproduce linear functions sampled at the vertices of $P$. Warren (1996) describes a construction for rational barycentric coordinate functions of degree $n-d$ where $d$ is the dimension of $P$ and $n$ is the number of facets of $P$. In this paper, we show that any rational coordinates functions of degree $n-d$ that are barycentric on $P$ are unique and of minimal degree.
\end{abstract}

\section{Introduction}

A convex polytope $P$ is the convex hull of a non-empty, finite set of points. If the set of points contains $d+1$ affinely independent points, then $P$ has dimension $d$. A $d$-simplex is the convex hull of $d+1$ affinely independent points. One of the primary facts concerning convex polytopes is the following (see [Gru67] for a proof):

THEOREM 1. A convex polytope can always be expressed as the intersection of a finite number of linear halfspaces.

$$
P=\bigcap_{i=1}^{n}\left\{x \mid H_{i}(x) \geq 0\right\} .
$$

The restriction of $P$ to the boundaries of a subset of the halfspaces is a face of $P$. A face of dimension zero is vertex of $P$. If $P$ is of dimension $d$, then a $(d-1)$ dimensional face of $P$ is a facet of $P$. A $k$-dimensional face $F$ of a $d$-dimensional polytope has co-dimension $d-k$.

\section{A characterization of barycentric coordinates}

Given a $d$-dimensional polytope $P$ in $\mathbb{R}^{d}$, let $v(P)$ denote the set of vertices of $P$. The rational functions $\left\{B_{v} \mid v \in v(P)\right\}$ are barycentric coordinates with respect to $P$ if the following properties are satisfied.

- Non-negativity - For all $v$ in $v(P)$,

$$
B_{v}(x) \geq 0 \quad \forall x \in P
$$

2000 Mathematics Subject Classification. Primary 52B11, 52B55; Secondary 52A38.

Key words and phrases. Barycentric coordinates, convex, polyhedra, polytopes.

(C) 2003 (American Mathematical Society) 
- Linear precision - Given a linear function $L(x)$,

$$
L(x)=\sum_{v \in v(P)} L(v) B_{v}(x) .
$$

If $L(x)=1$, then linear precision implies that the barycentric coordinates from a partition of unity; that is, they sum to one.

Warren [War96] gives a construction for rational barycentric coordinate functions for a convex polytope $P$. If $P$ is of dimension $d$ and bounded by $n$ facets, these coordinate functions are rational functions of the form

$$
B_{v}(x)=\frac{b_{v}(x)}{c(x)},
$$

where $b_{v}(x)$ is polynomial of degree $n-d$ and $c(x)$ is a polynomial of degree $n-d-1$. (A simple geometric construction for these coordinates in two dimensions appears in [Wach75, MLBD02] with a similar construction for three dimensions and higer appearing [WSDH03].)

One interesting question left unanswered by these papers is whether there exist other rational barycentric coordinate functions of lower degree. In this paper, we show that any set of rational coordinate functions of degree $n-d$ that satisfy the two properties above are unique and of minimal degree. The next section establishes the basic structure of barycentric coordinate functions. The second section explores the behavior of barycentric coordinate functions on the facets of $P$. The final section then shows that coordinate functions of degree $n-d$ are unique. An attached appendix proves several technical results associated with the theorems.

\section{The structure of barycentric coordinate functions}

In this section, we analyze the structure of barycentric coordinate functions based on the assumption that they are rational, non-negative and reproduce linear function over $P$. We first show that the numerator of the barycentric coordinate functions must be divisible by the equations of the facets of $P$.

Theorem 2. Let $H_{i}(x)=0$ define a facet $F$ of $P$. Then for all $v \notin v(F)$, $B_{v}(x)$ must vanish on $F$.

Proof. The vertex $v$ lies on $F$ exactly when $H_{i}(v)$ is zero. Consider the linear precision property with $H_{i}(x)$ in place of $L(x)$.

$$
\begin{aligned}
H_{i}(x) & =\sum_{v \in v(P)} H_{i}(v) B_{v}(x) . \\
& =\sum_{v \in v(P) \backslash v(F)} H_{i}(v) B_{v}(x) .
\end{aligned}
$$

For all $x$ in $F, H_{i}(x)$ vanishes so

$$
\sum_{v \in v(P) \backslash v(F)} H_{i}(v) B_{v}(x)=0
$$

for all $x \in F$. By construction, $H_{i}(v)$ must be strictly greater than zero for $v \notin v(F)$. Since $B_{v}(x)$ is non-negative over $F$, the $B_{v}(x)$ must be identically zero over $F$ for those $v \notin v(F)$. 
If the rational function $B_{v}(x)$ vanishes on $F$, then it also must vanish on $\left(H_{i}(x)=0\right)$. The numerator of $B_{v}(x)$, the polynomial $b_{v}(x)$ must also vanish on $\left(H_{i}(x)=0\right)$. By [Wal50, Ch.I, Theorem 9.7], the polynomial $H_{i}(x)$ must divide $b_{v}(x)$. Since $b_{v}(x)$ vanishes on those facets not containing $v, b_{v}(x)$ can be rewritten as

$$
b_{v}(x)=a_{v}(x) \prod_{j \notin i(v)} H_{j}(x),
$$

where $i(v)$ denotes the indices of those faces that contain $v$; that is,

$$
i(v)=\left\{j \mid H_{j}(v)=0\right\} .
$$

Note that non-negativity and linear precision determined part of the structure of $b_{v}(x)$. Surprisingly, these two properties also yield insight into the structure of $a_{v}(x)$. To understand this structure, we note that a polynomial $q(x)$ has a zero of multiplicity $\mathrm{k}$ at a point $x$ if $q$ and all of its partial derivatives up to and only up to order $k-1$ vanish at $x$. Due to non-negativity and linear precision, the denominator $c(x)$ for any barycentric coordinate function must have zeroes of a high multiplicity along certain faces. (See the appendix for a proof of the following theorem.)

TheOREM 3. Let $F$ be a face of co-dimension $k$ in $P$. If $F$ lies on exactly $m$ facets of $P, c(x)$ has a zero of multiplicity at least $m-k$ along $F$.

Note that $m-k \geq 0$ since a face of co-dimension $k$ always lies on at least $k$ facets. For example, the vertex of a square pyramid $(d=3)$ has four triangular faces $(m=4)$ meeting at common vertex. In this case, $c(x)$ has a zero of multiplicity one at the common vertex. More generally, if $|i(v)|$ denotes the numbers of facets containing a vertex $v, c(x)$ has multiplicity at least $|i(v)|-d$ at a vertex $v$.

We also claim that $b_{v}(x)$ has multiplicity $|i(v)|-d$ at a vertex $v$. (Again, see the appendix for a proof of the following theorem.)

THEOREM 4. If $c(x)$ has a zero of multiplicity $k$ at a vertex $v$ of $P$, then $b_{w}(x)$ has a zero of multiplicity at least $k$ at $v$ for all $w \in v(P)$.

Recall that $b_{v}(x)=a_{v}(x) \prod_{j \notin i(v)} H_{j}(x)$ with the $H_{j}(x)$ being non-zero at $v$. By the theorem, $b_{v}(x)$ has a zero of multiplicity $|i(v)|-d$ at $v$. If $b_{v}(x)$ is a polynomial of degree $n-d, a_{v}(x)$ is a polynomial of degree $|i(v)|-d$ and also has a zero of multiplicity $|i(v)|-d$ at $v$. In particular, $a_{v}(x)$ consists of the order $|i(v)|-d$ terms of the power series expansion of $b_{v}(x)$ at $v$. (If $v$ is the origin, $a_{v}(x)$ is a homogeneous polynomial with the zero set of $a_{v}(x)$ being a cone.)

For the remaining vertices $w \neq v, B_{w}(v)$ must be zero by Theorem 2. Since $B_{w}(x)=b_{w}(x) / c(x)$, the multiplicity of $b_{w}(x)$ at $v$ must be strictly greater than the multiplicity of $c(x)$ at $v,|i(v)|-d$.

\section{Facial behavior of barycentric coordinates}

We next show that if the barycentric coordinate functions are of degree $n-d$, their restriction to a facet $F$ of $P$ has degree $m-(d-1)$ where $m$ is the number of $(d-2)$-dimensional facets bounding $F$. This result is pleasing in that it suggests that if the barycentric coordinate functions have minimal degree, then the restrictions of these coordinate functions to the faces of $P$ also have minimal degree.

TheOREM 5. Let $\left\{B_{v}(x) \mid v \in v(P)\right\}$ be barycentric coordinate functions of degree $n-d$ for the polytope $P$. If $F$ is a facet of $P$ bounded by $m$ faces of dimension 
$d-2$, then the restriction of the functions $\left\{B_{v}(x) \mid v \in v(F)\right\}$ to $F$ are barycentric coordinate functions of degree $m-(d-1)$.

Proof. By Theorem 2, those $B_{v}(x)$ for which $v \notin v(F)$ must vanish on $F$. Since non-negativity and linear precision hold on $P$, they must also hold on the restriction to $F$. It remains to show that $\left\{B_{v}(x) \mid v \in v(F)\right\}$ restricted to $F$ have degree $m-(d-1)$.

If $F$ is bounded by $m$ faces of dimension $d-2$, there must exist $n-m-1$ remaining facets of $P$ that do not intersect $F$ in a $(d-2)$-dimensional face of $P$. Denote these facets $F_{1}, F_{2}, \ldots, F_{n-m-1}$. We will show that $c(x)$ and $b_{v}(x)$ vanish on

$$
E_{i}=h(F) \cap h\left(F_{i}\right)
$$

for all $1 \leq i \leq n-m-1$. Here, $h(F)$ denotes the affine hull of the face $F$.

Let $S_{i}$ be the intersection of the facet $F$ and the facet $F_{i}$. Since $F$ and $F_{i}$ do not intersect in a common facet, $h\left(S_{i}\right)$ is strictly an affine subspace of $E_{i}$. Let $L_{i}(x)=0$ be a supporting hyperplane containing $S_{i}$ that does not contain $E_{i}$. By linear precision,

$$
L_{i}(x) c(x)=\sum_{v \in v(P) \backslash v\left(S_{i}\right)} L_{i}(v) b_{v}(x) .
$$

If $v \in v(P) \backslash v\left(S_{i}\right)$, then either $v \in v(P) \backslash v(F)$ or $v \in v(P) \backslash v\left(F_{i}\right)$. For such $v$, $b_{v}(x)$ must vanish on either $h(F)$ or $h\left(F_{i}\right)$ by Theorem 2. Since the right-hand side vanishes on $E_{i}$, the left hand side must also vanish on $E_{i}$. Since $L_{i}(x)$ was chosen so as to not vanish on $E_{i}, c(x)$ must vanish on $E_{i}$.

We next show that if $v \in v\left(S_{i}\right)$, then $b_{v}(x)$ also vanishes on $E_{i}$. Since $c(x)$ vanishes on $E_{i}$,

$$
\sum_{v \in v\left(S_{i}\right)} b_{v}(x)=0
$$

on $E_{i}$. As observed in the last section, the order $|i(v)|-d$ terms of the power series expansion of $b_{v}(x)$ at $v$ are exactly $a_{v}(x)$. The remaining $b_{w}(x)$ for $w \neq v$ have a higher multiplicity at $v$. Since the sum of these functions vanishes on $E_{i}$, the order $|i(v)|-d$ terms of the power series expansion of this sum at $v$, namely $a_{v}(x)$, also must vanish along $E_{i}$. Therefore $b_{v}(x)$ also vanishes on $E_{i}$.

To conclude the proof, we note that both $b_{v}(x)$ and $c(x)$ vanish along $E_{i}$. Restricted to $h(F)$, each of the $E_{i}$ corresponds to a common linear factor of $b_{v}(x)$ and $c(x)$. Cancellation of these common factors decreases the degree of $B_{v}(x)$ by $n-m-1$ on $F$. Thus, the functions $B_{v}(x)$ restricted to $F$ are actually rational functions of degree $(n-d)-(n-m-1)=m-(d-1)$, the number of facets of $F$ minus its dimension.

Figure 1 illustrates an example of the theorem in the case of square pyramid. If $F$ is triangular face of the pyramid, then $F_{1}$ is the remaining triangular face that intersects the pyramid only at its apex. $S_{1}$ is the apex of the pyramid. $E_{1}$ the intersection of the plane containing $F$ and $F_{1}$. In this case, it is a line through the apex of the pyramid.

\section{Uniqueness of barycentric coordinates}

We are now ready to prove the main result of this paper, that rational barycentric coordinates of degree $n-d$ are unique and of minimal degree. This proof will procede by induction on the dimension of the polytope. 


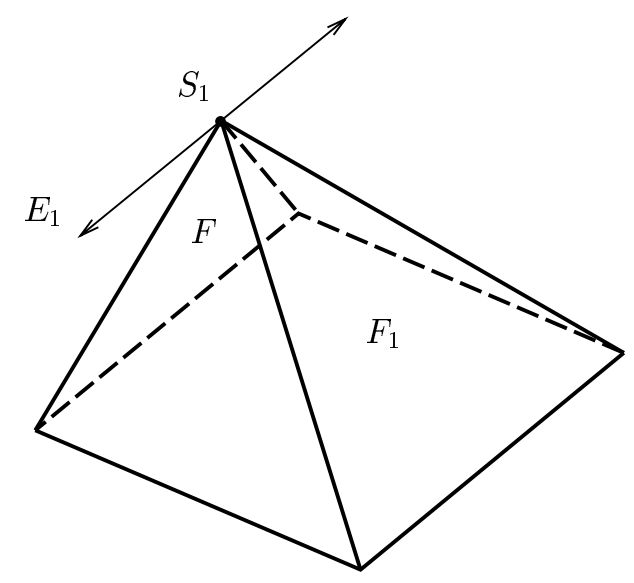

Figure 1. A labeled vertex of a square pyramid.

THEOREM 6. Let $\left\{B_{v}(x) \mid v \in v(P)\right\}$ be rational barycentric coordinates of degree $n-d$ for a polytope $P$ of dimension d bounded by $n$ facets. Then the coordinate functions $\left\{B_{v}(x) \mid v \in v(P)\right\}$ are unique and of minimal degree.

Proof. Let

$$
\left\{\frac{b_{v}(x)}{c(x)} \mid v \in v(P)\right\} \quad \text { and } \quad\left\{\frac{\hat{b}_{v}(x)}{\hat{c}(x)} \mid v \in v(P)\right\}
$$

be two sets of barycentric coordinate functions. We will show that for all $v \in P$,

$$
\begin{aligned}
b_{v}(x) & =\alpha \hat{b}_{v}(x), \\
c(x) & =\alpha \hat{c}(x) .
\end{aligned}
$$

The proof will proceed by induction on the dimension of the faces of $P$. On the edges of $P$, the two coordinate systems are barycentric by Theorem 5 . Thus, $b_{v}(x) / c(x)$ and $\hat{b}_{v}(x) / \hat{c}(x)$ must reduce to linear interpolation and therefore agree up to multiplication by a constant.

Assume the theorem holds for all facets of $P$. Since $b_{v}(x) / c(x)$ and $\hat{b}_{v}(x) / \hat{c}(x)$ are barycentric on the facets of $P$ by Theorem 5 , the coordinate functions must satisfy (by the inductive hypothesis)

$$
\begin{aligned}
b_{v}(x) & =\alpha_{i} \hat{b}_{v}(x), \\
c(x) & =\alpha_{i} \hat{c}(x),
\end{aligned}
$$

for each facet $F_{i}$ of $P$. Each pair of adjacent facets $F_{i}$ and $F_{j}$ must have $\alpha_{i}=\alpha_{j}$ along their intersection. Therefore, all facets share the same constant $\alpha$.

Since Equations (1) and (2) hold for all $n$ facets of $P$, the difference polynomials $b_{v}(x)-\alpha \hat{b}_{v}(x)$ and $c(x)-\alpha \hat{c}(x)$ vanish on the facets of $P$. Therefore, these difference polynomials must be divisible by the product of the $n$ linear equations defining these facets (see [Wal50, Ch. I, Theorem 9.7] for details). Since the coordinate functions are rationals of degree $n-d$, the difference polynomials must be identically zero everywhere and the coordinate functions agree everywhere. 
Note that the last part of the proof allows for the possibility of rational coordinates functions of degree $n$ (or greater) that are barycentric. Constructing such a wider class of such coordinates functions is an interesting problem for future research.

\section{Conclusion}

Barycentric coordinate have been always been know to exist for the tensor product of a set of simplices. The coordinate functions are simply the tensor product of barycentric coordinates for each of the simplices. This theorem establishes that the construction of Warren reproduces these functions in the case when $P$ is the tensor product of simplices.

The theorem also illustrates the power of the non-negativity and linear precision in building rational basis functions. Seemingly, simple properties can lead to powerful restrictions on the type of rational coordinate function that are allowable.

\section{References}

[Gru67] B. Grunbaum, Convex Polytopes, John Wiley and Sons, 1967

[Wal50] R. J. Walker, Algebraic Curves, Princeton, 1950.

[MLBD02] M. Meyer, H. Lee, A. Barr and M. Desbrun, Generalized barycentric coordinates for irregular polygons, Journal of Graphics Tools 7 (2002), 13-22.

[Wach75] E. Wachspress, A Rational Finite Element Basis, Academic Press, 1975.

[War96] J. Warren, Barycentric coordinates for convex polytopes, Advances in Computational Mathematics 6 (1996), 97-108.

[WSDH03] J. Warren, S. Schaefer, M. Desbrun, A. Hirani, Barycentric coordinates for convex sets, in preparation, 2003.

\section{Appendix}

In this appendix, we offer proofs of the last two theorems in the third section.

Theorem 3. Let $F$ be a face of co-dimension $k$ in $P$. If $F$ lies on exactly $m$ facets of $P, c(x)$ has a zero of multiplicity of at least $m-k$ along $F$.

Proof. The proof of Theorem 3 will procede by induction on the co-dimension $k$ of $F$. If $F$ has co-dimension zero i.e; $k=0$, then $F$ is the polytope $P$. Since $P$ lies on zero facets of itself, $m$ must be zero. Since $c(x)$ is non-zero, $c(x)$ has a zero of multiplicity 0 on $P$ and the theorem holds for $k=0$.

If the theorem holds for faces of co-dimension $k-1$, we next show that it holds for faces of co-dimension $k$. Let $F$ be a face of co-dimension $k$ lying on exactly $m$ common facets. Let $L(x)=0$ define a supporting hyperplane whose intersection with $P$ is exactly $F$. By linear precision,

$$
L(x) c(x)=\sum_{v \notin v(F)} L(v) b_{v}(x) .
$$

If $w \notin v(F)$, then let $\bar{F}$ be the smallest face containing $w$ and $F$. If $\bar{F}$ lies on $l$ of the $m$ facets containing $F$, then $b_{w}(x)$ has a zero of multiplity at least $l-(k-1)$ along $\bar{F}$ by induction. However, $b_{w}(x)$ also vanishes along the $m-l$ remaining facets that don't contain $\bar{F}$ by Theorem 2 . Since these facets also contain $F, b_{w}(x)$ has a multiplicity of at least $m-k+1$ along $F$. Therefore, by Equation (3), $c(x)$ has a zero of at least multiplicity $m-k$ along $F$. 
THEOREM 4. If $c(x)$ has a zero of multiplicity $k$ at a vertex $v$ of $P$, then $b_{w}(x)$ has a zero of multiplicity at least $k$ at $v$ for all $w \in v(P)$.

Proof. Recall that by partition of unity,

$$
c(x)=\sum_{v \in v(P)} b_{v}(x) .
$$

If $c(x)$ has multiplicity $k$ at a vertex $v$ of $P$, then assume that there exists a vertex $w$ such that $b_{w}(x)$ has a zero of multiplicity $l<k$ at $v$. Expand Equation (4) as a power series around $v . b_{w}(x)$ contains a set of terms of degree $l$. If the polynomial defined by these terms is negative on $P$ in some neighborhood of $v$, then $b_{w}(x)$ must also be negative on some neighborhood of $v$ in $P$. However, this violates the non-negativity of the $b_{w}(x)$ on $P$. If these terms are positive around $v$, then must exist another vertex $u$ whose order $l$ terms are negative around $v$. (Recall the expansion of $c(x)$ contains only terms of degree $k$ or higher.) Then, $b_{u}(x)$ violates non-negativity. Therefore, the $b_{w}(x)$ must have a zero of multiplicity $k$ at $v$ for all $w \in v(P)$.

Department of Computer Science, Rice University, Houston, Tx 77251

E-mail address: jwarren@rice.edu 